\title{
A RANDOMIZED DOUBLE BLIND STUDY COMPARING POST-OPERATIVE ANALGESIA PROVIDED BY MULTILEVEL THORACIC PARAVERTEBRAL BLOCK USING BUPIVACAINE (0.5\%) AND ROPIVACAINE (0.5\%) IN BREAST SURGERY
}

\author{
Kanchan Sharma ${ }^{1}$, Amit Agarwal2 ${ }^{2}$, Lalit Raiger ${ }^{3}$, Indra Kumari ${ }^{4}$
}

\section{HOW TO CITE THIS ARTICLE:}

Kanchan Sharma, Amit Agarwal, Lalit Raiger, Indra Kumari. "A randomized double blind study comparing postoperative analgesia provided by multilevel thoracic Para Vertebral Block using bupivacaine $(0.5 \%)$ and ropivacaine (0.5\%) in breast surgery". Journal of Evolution of Medical and Dental Sciences 2013; Vol2, Issue 34, August 26; Page: 6525-6532.

ABSTRACT: Paravertebral nerve block (PVB) is an anesthetic technique that results in less postoperative pain; nausea and vomiting require fewer analgesics. The aim of our study was to compare the postoperative analgesia provided by multilevel thoracic paravertebral injections of bupivacaine $(0.5 \%)$ and ropivacaine $(0.5 \%)$ in patients undergoing unilateral breast surgery. MATERIALS AND METHODS: It was a comparative randomized double blind study. Sample size was calculated at $80 \%$ study power and $\alpha$ error 0.05 came out to be 25 patients in each group which was further enhanced to 30 patients. Data were analyzed using SPSS version 12 . Student's T-test and Chisquare test were applied.60 ASA, I/ II patients, aged 18-56 years, body weight 50-80kg scheduled for unilateral breast surgery were randomly chosen to receive PVB from T2-T5 with either bupivacaine $0.5 \%$ (group 1 ) or ropivacaine $0.5 \%$ (group 2) $5 \mathrm{ml}$ at each level. Variables of efficacy were the amount of fentanyl consumption, postoperative pain on visual analogue pain score (VAS) upto 24 hours. Post-operative nausea and vomiting, any other complication, duration of block and both surgeon and patient's satisfaction score. RESULTS AND OBSERVATION: Time to rescue analgesic was 302.87 \pm 75.07 I group 1 and $299.89 \pm 86.67$ in group 2 and was statistically insignificant( P $>0.05$ ) However, mean duration of block was $21.21 \pm 5.521 \mathrm{hrs}$ and $22.63 \pm 3.874 \mathrm{hrs}$ in group 1 and in group 2 which was comparable.(P value $>0.05$ ). Seven patients in group 1 and four patients in group 2 required fentanyl as rescue analgesic. Frequency (times/patient) was $8(0.24 \pm .50)$ and $6(0.17 \pm .51)$ in group 1 and 2 respectively. Total dose of fentanyl in $\mathrm{mg} / \mathrm{kg} / \mathrm{patient}$ was $0.12 \pm 0.25$ and $0.09 \pm 0.26$ in group 1 and group 2 and is statistically insignificant. ( $P$ value $>0.05$ ). VAS score in the immediate post operative period was $1.12 \pm 0.48$ and $1.00 \pm .65$ in group 1 and group 2.(P $>0.05)$ VAS score at $4 \mathrm{hr}, 8 \mathrm{hr}, 12 \mathrm{hr}$ and at $24 \mathrm{hr}$ remained statistically insignificant. None of the patients in the PVB group had any complication in the first 24-hour in postoperative period. No statistical significances in postoperative nausea $(P=0.274)$, surgeon and patient's satisfaction score between the groups. CONCLUSION: Both drugs are equally effective in providing postoperative analgesia after breast surgery.

KEYWORDS: Unilateral breast surgery, multilevel PVB, bupivacaine $0.5 \%$ and ropivacaine $0.5 \%$,

INTRODUCTION: India faces a potential breast cancer epidemic over the next decade as women adopt western lifestyles by marrying and bearing children later in life, oncology experts say. The overall rate is now estimated at 80 new cases per 100000 population per year. ${ }^{1}$ 


\section{ORIGINAL ARTICLE}

Breast surgical procedures are typically performed using general anesthesia followed by inpatient hospitalization, but the side effects and complications such as postoperative nausea, vomiting and incisional pain which are all issues related more to the anesthesia, as opposed to operative experience and increases the hospital stay and thus the cost.

Postoperative pain continues to be under-treated. ${ }^{2}$ Patient controlled intravenous analgesia with opioids remains a common strategy for management of postoperative pain. ${ }^{3}$ However, this method is far from ideal because efficacy is suboptimal and side effects such as nausea, vomiting and sedation, are frequent.

Hence the search for alternative analgesic regimens in the perioperative period which may reduce the above mentioned unwanted side effects.

Paravertebral nerve block (PVB) is an anesthetic technique that can uniquely eliminate the cortical responses to thoracic dermatomal stimulation when compared with other forms of central block (e.g. epidural anesthesia). ${ }^{4}$ This block in addition to general anesthesia or supplemented with intravenous sedation only, seem to have shorter recovery times, experience less postoperative pain, require fewer analgesics, and experience less postoperative nausea and vomiting than breast surgery patients operated under general anesthesia without regional block.5, 6 Other than providing acute postoperative pain relief, pre -incisional PVB reduces the prevalence of chronic pain one year after breast surgery. ${ }^{7}$

The purpose of the present randomized study was to compare the efficacy of postoperative analgesia provided by multilevel thoracic PVB from level T2 through T5 using bupivacaine (0.5\%) as compared to ropivacaine $(0.5 \%)$ in patients undergoing breast surgery.

MATERIALS AND METHODS: Approval for the study was obtained from our institutional ethical committee. 60 female, ASA physical status I or II, aged 18-65 years, body weight 50-85 kg, scheduled for unilateral breast surgery, gave written informed consent to participate in this randomized, double-blinded study.

Primary exclusion criteria were: inability to provide informed consent, pregnancy, breast feeding, severe obesity (body mass index $>35 \mathrm{~kg} / \mathrm{m}^{2}$ ), coagulation disorders or treatment with anticoagulants, allergy to local anesthetics, severe spine or chest wall deformity and infection at the injection site, pathology involving axillary fold. A secondary exclusion criterion was surgical complication requiring re-operation during the study period. Sample size was calculated at $80 \%$ study power and $\alpha$ error 0.05 assuming a $30 \%$ increase in the duration of postoperative analgesia to be relevant. Thus sample size obtained was 25 patients in each group which was further enhanced to 30 patients assuming the possibility of dropouts.

Patients were randomized, by an opaque sealed envelope into Group 1 (0.5\% bupivacaine) and Group 2 (0.5\% ropivacaine) to receive multilevel thoracic PVB block. One day before the surgery patients were thoroughly explained about the procedure and its risks and benefits. They were thoroughly explained about the use of visual analogue scale and on demand analgesia for the postoperative pain. Patients were advised pre-operative fasting for a period of $6 \mathrm{~h}$ and were premedicated with tab. midazolam $7.5 \mathrm{mg}$ the night before the surgery.

On arrival to the operation theater they were taken to the monitored pre-operative holding area maintenance infusion of Ringer lactate was started through the 18G cannula in the contralateral limb. Patients were sedated by using $1 \mu \mathrm{g} / \mathrm{kg}$ of fentanyl and $1 \mathrm{mg}$ of midazolam 


\section{ORIGINAL ARTICLE}

intravenously. After standard monitoring: ECG, noninvasive arterial blood pressure (NIBP-Mean BP), pulse oximetry (SPO2), multilevel thoracic PVB was performed with the patient in sitting position under aseptic condition by using the classical loss of resistance technique. Patients were sitting with their neck flexed, back arched and shoulders dropped forward. The spinous process of T2-T5 was identified and a mark was placed at its most superior aspect. From the midpoint of these marks a needle entry site was marked $2.5 \mathrm{~cm}$ lateral to each spinous process ipsilateral to the incision. The

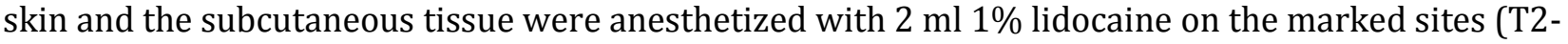
T5; four injections).

We used 22-gauge Quincke spinal needle for block, the shaft of which was grasped by the dominant hand of the anesthesiologist was inserted through the skin wheal and it was advanced anteriorly in the parasagittal plane (perpendicular to the back in all the directions) until it contacted the transverse process, at $2-5 \mathrm{~cm}$ depth, depending on the body habitus of the patient. As a safety measure, to prevent inadvertent depth placement we grasped the needle at a point from its tip that was equal to the estimated depth from the skin to the transverse process. Inserting the needle $1 \mathrm{~cm}$ past this predicted depth was allowed. If the transverse process was not identified at an appropriate depth, it was assumed that the needle tip was lying between the two transverse processes; the needle was then redirected cephalad and then caudal until the transverse process was successfully contacted. This depth was noted as the estimated distance to subsequent transverse processes. The needle was then withdrawn to the subcutaneous tissue and angled to walk off the caudal edge of the transverse process $1 \mathrm{~cm}$. Loss of resistance or a subtle "pop" was felt as the needle passed through the superior costotransverse ligament. After negative aspiration, $5 \mathrm{ml}$ of the drug according to the group was administered per site. The time of onset of sensory loss was assessed at $5 \mathrm{mins}$ and every 5 min thereafter upto 30 min after injection. A block was considered as failed if inadequate sensory block was not achieved after $30 \mathrm{~min}$ and that case was excluded from the study. The patients were shifted to operation theatre after $30 \mathrm{mins}$. The patient received a standardized anesthetic technique consisting of propofol (2-3 mg/kg) and fentanyl (50-100 $\mu \mathrm{g}$ ) for induction. After loss of eye reflex, a laryngeal mask airway (LMA, No. 3-4 Portex) was inserted and the patient's lungs were ventilated with $40 \%$ oxygen in air. Monitoring was continued throughout the operative procedure. Anesthesia was maintained with propofol, and boluses of fentanyl (50-100 $\mu \mathrm{g}$ ) I.V. were given if the heart rate and mean blood pressure were increased more than $20 \%$ from baseline. As a prophylactic antiemetic, all patients were treated with $4 \mathrm{mg}$ of Ondansetron I.V., 30 minutes before the end of the procedure.

Immediately after the operation the surgeons were asked to rate conditions in the operation field. (NRS 0-100).Parameters of the cardiovascular system [pulse rate (PR), mean blood pressure (BP) and oxygen saturation $\left.\left(\mathrm{SpO}_{2}\right)\right]$ before anesthesia, intra-operative and in the postoperative period every $4^{\text {th }}$ hourly for $24 \mathrm{~h}$ were analyzed. Patients were shifted to postoperative surgical ward. Patients were asked to record their pain by using visual analogue pain scale (VAS; $0=$ no pain to $10=$ worst pain imaginable) at rest and during a standardized movement (folding hands, moving both arms to an angle of $90^{\circ}$ to the body) every $4^{\text {th }}$ hourly for 24 hours. Analgesic treatment consisted of on demand fentanyl $0.2-0.5 \mu \mathrm{g} / \mathrm{kg}$ I.V. repeated every 5 minutes as necessary to relieve pain rated by using visual analogue pain scale (VAS $\geq 3$ ). Antiemetic ondansetron $4 \mathrm{mg}$ I.V. was administered if the patient complained of nausea. Time to first rescue analgesia, duration of analgesia (time between the last suture application and the demand for first rescue analgesic), the doses of all the drugs and their 
times of administration, as well as any other problems or complications were noted down. 24 hours after the operation the patients were asked to rate their whole experience of anesthesia with multilevel thoracic paravertebral block. (NRS 0-100)

The primary outcome measure was the cumulative consumption of intravenous fentanyl over 24 hours in both the groups.

RESULTS: 60 patients were randomized to paravertebral injections with bupivacaine $(n=30)$ or ropivacaine $(n=30)$.one patient from bupivacaine group was re-operated during the study period and was excluded from the study, thus data from 59 patients (bupivacaine, $n=29$; or ropivacaine $n=$ 30) remained for analysis. Data collection was done with the help of semi-structured pretested proforma and observation of the patients postoperatively every $4^{\text {th }}$ hourly up to 24 hours postoperatively in the ward. Data entry was done by using MS-Excel and were analyzed using the software SPSS for windows (version 12.0, SPSS Inc., Chicago, IL). Student's T-test and Chi-square test was applied. Results were presented as mean \pm sd or no. of patients (percent); P value $<0.05$ defined statistical significant difference.

Patients in both the study groups were not different regarding their initial characteristic data. (Table 1)

Table 1: Demographic variables and Pre-operative parameters

\begin{tabular}{|c|c|c|c|}
\hline Variables and Parameters & Group 1(n=29) & Group 2(n=30) & P value \\
\hline Age (years) & $42.67 \pm 10.50$ & $40.26 \pm 11.10$ & 0.94 \\
\hline Weight (kg) & $51.39 \pm 4.26$ & $52.46 \pm 4.736$ & 0.58 \\
\hline Pulse rate (bpm) & $76.27 \pm 7.25$ & $78.37 \pm 6.45$ & 0.30 \\
\hline Mean arterial pressure (mm Hg) & $90.91 \pm 4.64$ & $91.11 \pm 9.36$ & 0.13 \\
\hline SPO2 (\%) & $99.04 \pm 0.84$ & $99.10 \pm 0.85$ & 0.75 \\
\hline
\end{tabular}

Data are given as mean \pm sd, Test applied: $T$ test, $n$ : number of patients,

Bpm: Beats per minute

Haemodynamic parameters and oxygen saturation values were similar in both the groups during the whole perioperative period (Table $1 \& 2$ ).

Table 2- Intra-operative variables and parameters

\begin{tabular}{|c|c|c|c|}
\hline Variables and Parameters & Group 1(n=29) & Group 2(n=30) & P value \\
\hline Pulse rate (bpm) & $78.22 \pm 6.04$ & $77.40 \pm 5.29$ & 0.83 \\
\hline $\begin{array}{c}\text { Mean arterial pressure } \\
(\mathrm{mm} \mathrm{Hg)}\end{array}$ & $93.44 \pm 23.85$ & $95.39 \pm 18.64$ & 0.72 \\
\hline SPO2 (\%) & $99.03 \pm 0.72$ & $99.10 \pm 0.65$ & 0.75 \\
\hline IF ( $\mu \mathrm{g} / \mathrm{kg})$ & $102.27 \pm 10.76$ & $103.29 \pm 12.48$ & 0.362 \\
\hline $\begin{array}{c}\text { Surgeon satisfaction score } \\
\text { NRS (0-100) }\end{array}$ & $80.58 \pm 8.29$ & $79.77 \pm 9.50$ & 0.73 \\
\hline
\end{tabular}

Data are given as mean \pm sd, Test applied: $T$ test, $n$ : number of patients, bpm Beats per minute, IF intra-operative fentanyl, NRS: numeric rating scale. 
The total dose of propofol in group 1 and group 2 was $218.67 \pm 12.66$ and $220.52 \pm 11.56$ which was statistically insignificant. $(\mathrm{P}>0.05)$ Time to request for analgesic for the first time was considered as the duration of block and hence post-operative analgesia which was 302.87 \pm 75.07 I group 1 and $299.89 \pm 86.67$ in group 2 and was statistically insignificant $(\mathrm{P}>0.05)$ However, mean duration of block was $21.21 \pm 5.521 \mathrm{hrs}$ and $22.63 \pm 3.874 \mathrm{hrs}$ in group 1 and in group 2 respectively; which was comparable. . (P value >0.05). Seven patients in group 1 and four patients in group 2 required fentanyl as rescue analgesic. Frequency (times/patient) was $8(0.24 \pm .50)$ and $6(0.17 \pm .51)$ in group 1 and 2 respectively. Total dose of fentanyl in $\mathrm{mg} / \mathrm{kg} /$ patient was $0.12 \pm 0.25$ and $0.09 \pm 0.26$ in group 1 and group 2 and is statistically insignificant. (P value $>0.05$ ). VAS score in the immediate post operative period was $1.12 \pm 0.48$ and $1.00 \pm .65$ in group 1 and group 2.(P > 0.05)VAS score at $4 \mathrm{hr}, 8 \mathrm{hr}, 12 \mathrm{hr}$ and at $24 \mathrm{hr}$ remained statistically insignificant. 11\%patient in group 1 and $10.50 \%$ in group 2 having PONV required treatment .None of the patients in the PVB group had any complication in the first 24-hour in postoperative period.

\section{Table 3: Post -operative characteristics}

\begin{tabular}{|c|c|c|c|}
\hline Variables & Group 1(n=29) & Group 2(n=30) & P value \\
\hline VAS score at 4hrs & $4.24 \pm 0.58$ & $4.67 \pm 0.88$ & 0.06 \\
\hline VAS score at 8hrs & $2.55 \pm 0.63$ & $2.53 \pm 0.62$ & 0.11 \\
\hline VAS score at 12hrs & $2.87 \pm 0.39$ & $2.78 \pm 0.54$ & 0.55 \\
\hline VAS score at 24hrs & $2.69 \pm 0.60$ & $2.64 \pm 0.36$ & 0.23 \\
\hline VAS score at first rescue analgesic & $4.34 \pm 0.68$ & $4.57 \pm 0.77$ & 0.06 \\
\hline Maximum VAS score in 24hrs & $4.62 \pm 0.88$ & $4.58 \pm 0.77$ & 0.35 \\
\hline Patient satisfaction score NRS(0-100) & $79.47 \pm 9.17$ & $78.76 \pm 8.48$ & 0.73 \\
\hline
\end{tabular}

Data are given as mean \pm sd, Test applied: $T$ test, $n$ : number of patients, Bpm: Beats per minute, VAS: visual analogue scale, NRS: numeric rating scale

\section{Comparison of maximum VAS score In $24 \mathrm{hrs}$ in both the Groups}

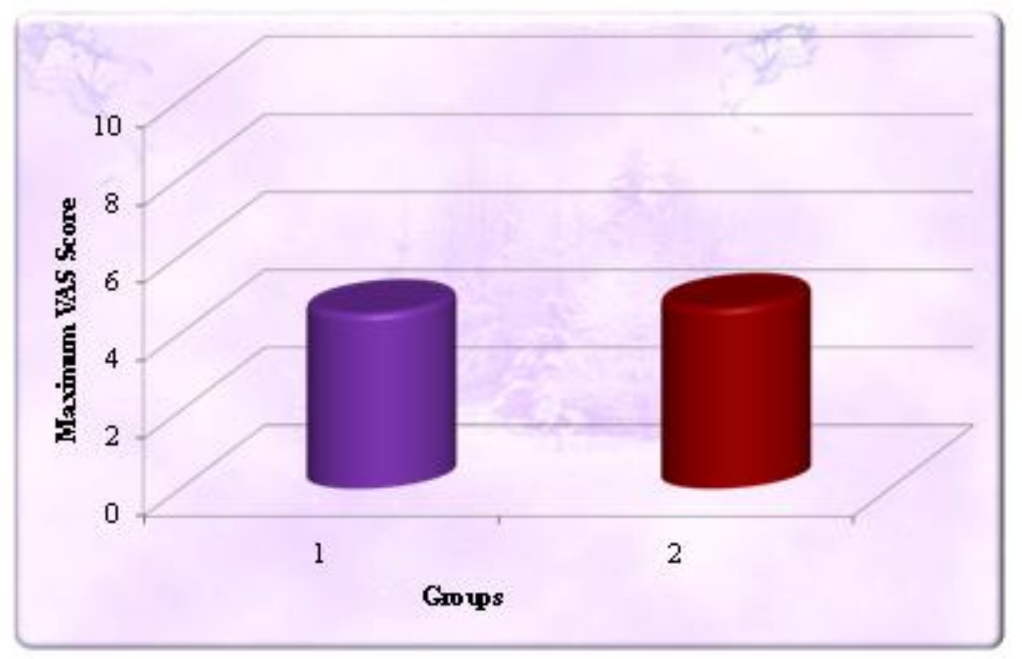




\section{ORIGINAL ARTICLE}

\section{Comparison of the time to first rescue Analgesic at vas $\geq 3$ in both the groups}

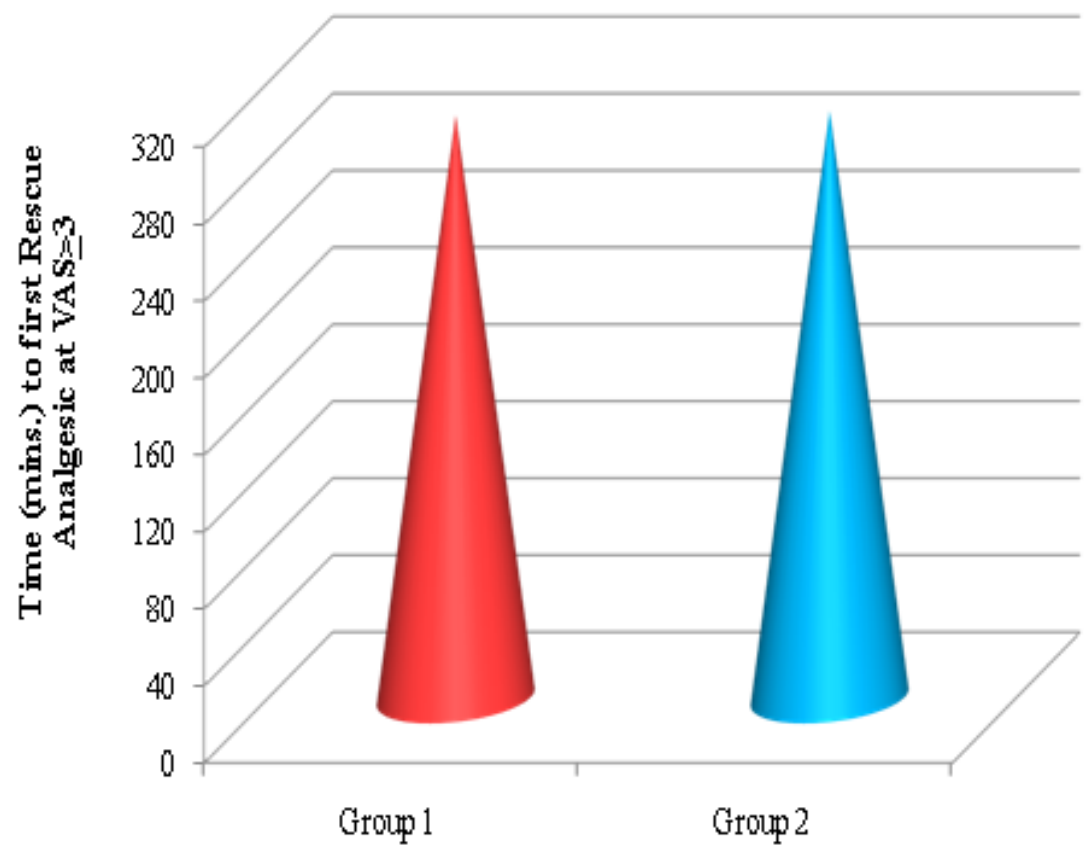

DISCUSSION: In the current medical literature there are no clinical trials comparing multilevel thoracic paravertebral using ropivacaine $(0.5 \%)$ and bupivacaine $(0.5 \%)$ in patients scheduled for various breast surgeries, so there are not direct comparisons to the present study results obtained with other studies in the discussion section.

In our study paravertebral block using ropivacaine $(0.5 \%)$ and bupivacaine $(0.5 \%)$ produced comparable analgesia which persisted for 24 hours in most of the patients. We were able to confirm these facts based on the similar consumption of postoperative fentanyl requirement as well as less pain score on VAS $\leq 3$.

Most of the time taken for performance of any regional anesthetic procedure is consumed in identifying the relevant anatomy, preparing the area and loading the local anesthetic drugs. This is definitely a disadvantage for a technique that requires positioning and multiple injections. However we have used a monitored preoperative holding area to place the blocks to avoid this problem. This might explain the lack of anticipated delay in performance of four injections technique in our study, as the performance of the block is accomplished quickly.

With respect to the technique itself, there are several approaches to achieve the block. Both single ${ }^{8,9}$ and multilevel 10,11 injections have been reported to provide good analgesia. The single puncture technique provides more patient comfort and lowers the need for sedation during the procedure, thereby improves the patient satisfaction. ${ }^{12}$ But on the other hand inadvertent injection of a larger volume of local anesthetic is more risky than the multiple injections of small volume .On the contrary multilevel injection produces a more reliable sensory block than a single injection technique and assessment of longitudinal anesthetic spread in paravertebral space revealed a more caudal spread of local anesthetic compared with a cephalic distribution .11We have reduced the discomfort of multiple injections to the patient by giving midazolam, fentanyl, and local infiltration of skin and subcutaneous tissue before the placement of block. 


\section{ORIGINAL ARTICLE}

The incidence of PONV in our patient population was relatively infrequent, considering that the general risk of PONV in women undergoing breast surgery under general anesthesia is high. The less PONV may be associated with improved analgesia and less postoperative fentanyl consumption.

The limitation of our study was that we did not compare our study groups to a placebo group. Therefore, we were not able to compare the advantages (better postoperative analgesia, less PONV, less opioids consumption) of combining regional anesthesia (PVB) with GA v/s patients given GA alone. We limited our period of observation up to $24 \mathrm{~h}$ only, so our study did not show postoperative analgesia with PVB to be prolonged as shown by Klein ${ }^{6}$ and colleagues, who found reduced postoperative pain score at $72 \mathrm{~h}$.

To conclude this study suggested that both bupivacaine $(0.5 \%)$ and ropivacaine $(0.5 \%)$ provided equally effective analgesia of same duration, equally less postoperative nausea and good patient satisfaction score after a multilevel thoracic paravertebral block. This suggests that both drugs are equally effective in providing postoperative analgesia after breast surgery.

\section{REFERENCES:}

1. Bagchi S, Kolkata, India. Breast Cancer Rises in India Current Science 2001; 81:465-74.

2. Poleshuck EL, Katz J, Andrus CH, Hogan LA, Jung BF, Kulick DI, et al. Risk factors for chronic pain following breast cancer surgery: a prospective study. J Pain 2006;7:626-34

3. Macintyre PE. Safety and efficacy of patient controlled analgesia. Br J Anaesth 2001; 87:3646.

4. Richardson J, Jones J, Atkinson R. The effect of thoracic paravertebral blockade. Anesth Analg 1998; 87:373-6.

5. Pusch F, Freitag H, Weinstable C, Obwegeser R, Huber E, Wildling E. Single-injection paravertebral block compared to general anaesthesia in breast surgery. Acta Anaesthesiol Scand 1999; 43:770-4.

6. Klien SM, Bergh A, Steeel SM, Georgiade GS, Greengrass RA Thoracic Paravertebral block for breast surgery. Anesth Analg 2000; 90:1402-5.

7. Kairaluoma PM, Bachman MS, Pere PJ, Rosenberg PH. Pre incisional paravertebral block reduce prevalence of chronic pain after breast surgery. Anesth Analg 2006; 103:703-8

8. Kairaluoma PM, Bachman MS, Korpinen AK, Rosenberg PH, Pare PJ. Single injection paravertebral block before general anesthesia enhances analgesia after breast cancer surgery with and without associated lymph node biopsy. Anesth Analg 2004; 99:1837-43.

9. Moller JF, Nikolajsen L, Rodt SA, Ronning H, Carlsson PS.Thoracic paravertebral block for breast cancer surgery: A randomized double-blind study. Anesth Analg 2007; 105:1848-51.

10. Coveney E, Weltz CR, Greenglass R, Iglehart D, Leight GS, Steele SM, Lyerly HK Use of paravertebral block anesthesia in the surgical management of breast cancer. Ann Surg 1998; 227:496-501.

11. Naja ZM, El-Rajab M, AI-Tannir MA, Ziade FM, Tayara K, Younes F, Lonnqist PA Thoracic paravertebral block: influences the number of injections Reg Anesth Pain Med 2006 ;31:196201

12. Dabbagh A, Elyasi H: The role of paravertebral block in decreasing postoperative pain in elective breast surgeries. Med Sci Monit, 2007; 13: CR464-7 


\section{ORIGINAL ARTICLE}

\section{AUTHORS:}

1. Kanchan Sharma

2. Amit Agarwal

3. Lalit Raiger

4. Indra Kumari

\section{PARTICULARS OF CONTRIBUTORS:}

1. Senior Resident, Department of Anaesthesia, SMS Medical College, Jaipur.

2. Junior Consultant, Department of Anaesthesia, Nova Group.

3. Professor, Department of Anaesthesia, R.N.T. Medical College, Udaipur.
4. Professor and HOD, Department of Anaesthesia, R.N.T. Medical College, Udaipur.

NAME ADDRESS EMAIL ID OF THE CORRESPONDING AUTHOR:

Dr. Kanchan Sharma, 3/96, Vidhyadhar Nagar, Jaipur.

Email-drkanchanamit@gmail.com

Date of Submission: 09/08/2013.

Date of Peer Review: 10/08/2013.

Date of Acceptance: 20/08/2013.

Date of Publishing: 23/08/2013 